\title{
An Italian consensus on pulmonary rehabilitation in COVID-19 patients recovering from acute respiratory failure: results of a Delphi process
}

\author{
Michele Vitacca ${ }^{1}$, Marta Lazzeri ${ }^{2,3}$, Enrico Guffanti ${ }^{4}$, Pamela Frigerio ${ }^{5}$, Francesco D'Abrosca ${ }^{3}$, \\ Silvia Gianola ${ }^{6}$, Mauro Carone ${ }^{1}$, Mara Paneroni ${ }^{1,3}$, Piero Ceriana ${ }^{1}$, Franco Pasqua ${ }^{7}$, Paolo Banfi ${ }^{8}$, \\ Francesco Gigliotti ${ }^{9}$, Carla Simonelli ${ }^{1}$, Serena Cirio ${ }^{1}$, Veronica Rossi ${ }^{10}$, Chiara G. Beccaluva ${ }^{11}$, \\ Mariangela Retucci ${ }^{12}$, Martina Santambrogio ${ }^{3,12}$, Andrea Lanza ${ }^{3,13}$, Francesca Gallo ${ }^{12}$, \\ Alessia Fumagalli ${ }^{4}$, Marco Mantero ${ }^{12,14}$, Greta Castellini' ${ }^{6}$, Mariaconsiglia Calabrese ${ }^{15,16}$, \\ Giorgio Castellana $^{1}$, Eleonora Volpato ${ }^{8}$, Marina Ciriello ${ }^{16,17}$, Marina Garofano ${ }^{16}$, Enrico Clini ${ }^{18}$, \\ Nicolino Ambrosino ${ }^{1}$ on behalf of AIPO (Associazione Italiana Pneumologi Ospedalieri), \\ ARIR (Associazione Riabilitatori dell'Insufficienza Respiratoria), SIP (Società Italiana di Pneumologia) \\ AIFI (Associazione Italiana Fisioterapisti) and SIFIR (Società Italiana di Fisioterapia e Riabilitazione)
}

${ }^{1}$ Pulmonary Rehabilitation Units, ICS Maugeri IRCCS, Pavia; ${ }^{2}$ Department of Cardiothoracic and Vascular Surgery, ASST Grande Ospedale Metropolitano Niguarda, Milan; ${ }^{3}$ Italian Association of Respiratory Physiotherapists (ARIR) Unità Spinale ASST, Grande Ospedale Metropolitano Niguarda, Milan; ${ }^{4}$ Pulmonary Rehabilitation Unit, Italian National Research on Aging (IRCCS INRCA), Casatenovo (LC); ${ }^{5}$ Maternal and Pediatric Department, ASST Grande Ospedale Metropolitano Niguarda, Milan; ${ }^{6}$ Unit of Clinical Epidemiology, IRCCS Istituto Ortopedico Galeazzi, Milan; ${ }^{7}$ Pulmonary Rehabilitation Unit, Clinical Rehabilitation Institute of Villa delle Querce, Nemi (RM); ${ }^{8}$ Pulmonary Rehabilitation Unit, Fondazione Don Carlo Gnocchi, Milan; ${ }^{9}$ Pulmonary Rehabilitation Unit, Fondazione Don Carlo Gnocchi, Florence; ${ }^{10}$ Health Professions Department Unit, Fondazione IRCCS Ca' Granda Ospedale Maggiore Policlinico, Milan; ${ }^{11}$ Pulmonary Rehabilitation Unit, ASST Ospedale Maggiore, Crema; ${ }^{12}$ Respiratory Unit and Cystic Fibrosis Adult Center, Fondazione IRCCS Ca' Granda Ospedale Maggiore Policlinico, Milan; ${ }^{13}$ Department of Neuroscience, Sleep Medicine Center, ASST Grande Ospedale Metropolitano Niguarda,

Correspondence: Michele Vitacca, ICS Maugeri IRCCS, Department of Respiratory Rehabilitation, Via S. Maugeri 4, 27100 Pavia, Italy. E-mail: michele.vitacca@icsmaugeri.it

Key words: COVID-19; SARS-CoV-2; physiotherapy; exercise; dyspnoea; pandemics; Delphi process.

Conflicts of interest: None for all authors

Funding: No funding was paid to the authors or their institutions for this work.

Acknowledgments: Thanks to the panelists: Ana Balana (Spain), Bruno Balbi (Italy), Stefano Belli (Italy), Raffaella Bellini (Italy), Annalisa Carlucci (Italy), Cesare Del Monaco (Italy), Vilma Donizetti (Italy), Giuseppe Gaudiello (Italy), Roger Goldstein (USA), Giuseppe Lapiana (Italy), Alda Marques (Portugal), Linda Nici (USA), Paola Pierucci (Italy), Emilia Privitera (Italy), Maurizio Sommariva (Italy), Paolo Tarsia (Italy), Ludovico Trianni (Italy), Ioannis Vogiatzis (UK), João Carlos Winck (Portugal), Elisabetta Zampogna (Italy).

Availability of data: Research data are stored in OSF repository (https://osf.io/8xcnd)

Received for publication: 9 June 2020 .

Accepted for publication: 9 June 2020.

${ }^{\circ}$ Copyright: the Author(s), 2020

Licensee PAGEPress, Italy

Monaldi Archives for Chest Disease 2020; 90:1444

doi: 10.4081/monaldi.2020.1444

This article is distributed under the terms of the Creative Commons Attribution Noncommercial License (by-nc 4.0) which permits any noncommercial use, distribution, and reproduction in any medium, provided the original author(s) and source are credited.
Milan; ${ }^{14}$ Department of Pathophysiology and Transplantation, University of Milan; ${ }^{15} \mathrm{AOU}$ San Giovanni di Dio e Ruggi d'Aragona, University of Salerno; ${ }^{16}$ Società Italiana Fisioterapia e Riabilitazione (S.I.Fi.R.); ${ }^{17}$ Monaldi Hospital, A.O.R.N. dei Colli, Naples; ${ }^{18}$ Department of Medical and Surgical Sciences of Mothers, Children and Adults, University of Modena and Reggio Emilia, Modena, Italy

\section{Abstract}

There is a need of consensus about the pulmonary rehabilitation (PR) in patients with COVID-19 after discharge from acute care. To facilitate the knowledge of the evidence and its translation into practice, we developed suggestions based on experts' opinion. A steering committee identified areas and questions sent to experts. Other international experts participated to a RAND Delphi method in reaching consensus and proposing further suggestions. Strong agreement in suggestions was defined when the mean agreement was $>7(1=$ no agreement and $9=$ maximal agreement). Panelists response rate was $>95 \%$. Twenty-three questions from 4 areas: personnel protection equipment, phenotypes, assessments, interventions, were identified and experts answered with 121 suggestions, 119 of which received high level of concordance. The evidence-based suggestions provide the clinicians with current evidence and clinical experts opinion. This framework can be used to facilitate clinical decision making within the context of the individual patient. Further studies will evaluate the clinical usefulness of these suggestions. 


\section{Introduction}

The COVID-19 pandemic "day after" is coming and people, who suffered from mild to severe pneumonia up to hypoxaemic respiratory failure, might be at risk of long-term impairment and disability [1], which at present, are only speculative and need prospective studies. However, like patients undergone critical illnesses, these patients can present dyspnea and fatigue at rest and during activities of daily living (ADL), disability, exercise intolerance, reduction in peripheral muscle function and in nutritional status with significant weight loss. Furthermore they may be at risk of residual or evolutive parenchymal damage with respiratory muscle function impairment. Besides lung function the infection can negatively affect also other organs like heart, kidneys, muscles and brain, with significant health impacts that may persist. Additionally, people requiring intensive care are at increased risk for post-traumatic stress disorder (PTSD), anxiety, and depression $[2,3]$.

The modalities and impact of physiotherapy, the tolerance to and the results of pulmonary rehabilitation (PR) programs in these patients remain to be elucidated. To facilitate knowledge, translation and implementation of respiratory physiotherapy and PR of COVID-19 patients after acute illness, the Italian societies of respiratory healthcare professionals, AIPO (Associazione Italiana Pneumologi Ospedalieri), ARIR (Associazione Riabilitatori dell'Insufficienza Respiratoria), SIP (Società Italiana di Pneumologia) AIFI (Associazione Italiana Fisioterapisti) and SIFIR (Società Italiana di Fisioterapia e Riabilitazione) promoted a multidisciplinary international consensus, through a Delphi process, the results of which are reported in this paper.

\section{Methods}

\section{Phase 0}

In April 2020 the steering committee identified issues and posed questions to authors who had to answer with suggestions. Other 20 international experts (panelists) were invited to participate to a RAND Delphi method in reaching consensus and in proposing additional suggestions. A RAND modified rapid Delphi process was used [4] following the "Guidance for Developers of Health Research Reporting Guidelines" [5].

\section{Phase I: Review evidence and selection of items}

The steering committee included 3 physiotherapists, 11 respiratory physiotherapists, 12 pulmonologists, 2 psychologist and 2 methodologists (Figure 1A). To support the external validity of the consensus process, the steering committee had to be advocated among active members of their corresponding scientific association; additionally, they had to reflect the expertise required in PR and direct experience with the management of these patients.

The following research issues were identified:

1. personnel protection equipment (PPE)

2. phenotypes

3. assessments

4. interventions

Topics for each research area were submitted to experts providing suggestions, comments and references. Two settings were evaluated: post-acute hospital and home. Search results were shared and discussed by the steering committee and agreed suggestions were stated for each topic.

\section{Literature search}

A scoping review was conducted following the methodological framework by Arksey and O'Malley [6]. We comprehensively searched databases and other sources to identify literature on COVID-19 between $1^{\text {st }}$ December 2019 and $6^{\text {th }}$ April 2020. We performed a systematic search of MEDLINE via PubMed. Because of potential delays in indexing of databases, we also searched Google Scholar, the WHO official websites [8] and reference lists of the identified articles to find reports of additional studies. Experts formulated items ensuring that all potentially relevant questions would be included in the initial list of topics for the first Delphi round.

\section{Phase II: Selection of voting panel and Delphi rounds}

To ensure content validity, 20 international panelists (10 respiratory physiotherapists and 10 pulmonologists from 5 countries) participated to a two-round electronic Delphi process

\section{A} QUESTIONER'S PANEL

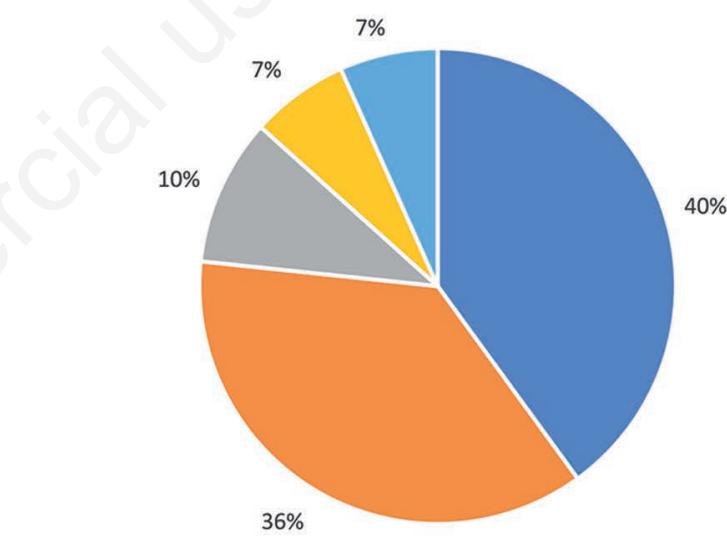

- pulmonologists " respiratory physiotherapists " physiotherapists " methodologists " psychologist

\section{INTERNATIONAL PANEL EXPERTS}

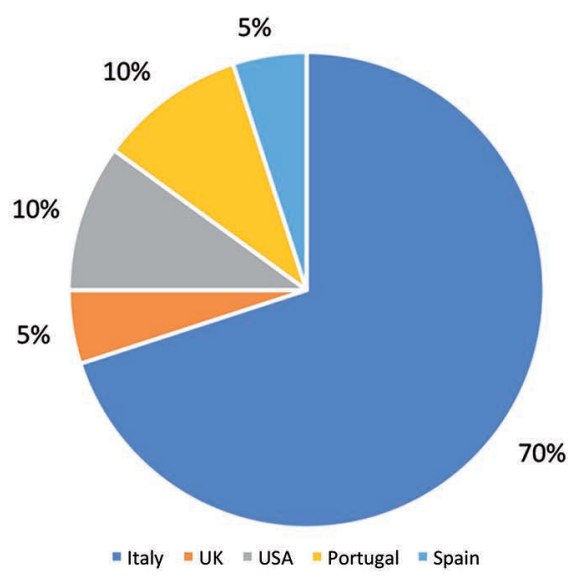

Figure 1. Distribution of steering committee authors (A) and panellists (B). 
(Figure 1B). The data collection was distributed via a web-based survey platform in Survey Monkey. For each recommendation, panelists had to rate the level of their agreement on a nine-point Likert scale, (1-3 = highly inappropriate with low agreement, 4$6=$ undecided with insufficient agreement, 7-9 = highly appropriate with high agreement). A free text-box was available and respondents were encouraged to comment their choices, regardless of rating. Panelists' rating and feedback data were exported from Survey Monkey as a de-identified data set into Microsoft Excel.

\section{Round 1}

Panelists were encouraged to use the full range of the ninepoint Likert scale and were asked to consider the agreement for each recommendation according to the settings. Up to two email reminders were sent to request completion.

\section{Round 2}

Following reception of all first-round data, reports were provided to each panelist illustrating the distribution of responses via a resume table, a reminder of their own first-round responses, a thematic summary of free-text responses and representative quotations providing panelists' perspectives regarding the specified suggestions. Panelists were asked to re-rate the consensus building process only for items requiring clarification or without agreement. Second-round items included suggestions categorized as having uncertain agreement by either panel in the first round and new suggestions by panelists in the first round of free-text responses. Up to two email reminders were sent to request completion.

\section{Final criteria list}

On the basis of the Delphi procedure, the final list of suggestions was composed. Items were modified, if necessary, by the steering committee according to the input of the participants in the Delphi rounds and the comments received during the process. The panelists' comments and the final set of suggestions "approved" and "not approved" were sent to the voting panel as closing audit procedure.

\section{Data analysis}

Following reception of responses from both rounds, we calculated agreement between panellists. We used the Kendall's W coef- ficient of concordance. The value of $\mathrm{W}$ ranges from 0 to 1 , with 0 indicating no consensus, and 1 indicating perfect consensus between lists. Therefore, we considered every single recommendation approved when a satisfactory agreement for $\mathrm{W}>0.7$. Items with W lower than 0.7 were submitted again. Disagreement was defined as at least $0.3(30 \%)$ of the scores being assigned to the bottom (1-3) and top (7-9) tertiles.

\section{Results}

\section{Phase I: evidence review}

The largest proportion (67.7\%) of published articles are from China, primarily exploring epidemiology, causes (38.5\%), clinical manifestations and diagnosis, as well as prevention and control of pandemic. Currently, there is no randomized controlled or cohort studies of PR programs for these patients; the recommended measures are supportive [8].

\section{Phase II: Delphi rounds}

\section{Round 1}

Among panellist, 100\% completed the first round. A total of 23 questions with 121 items were included. Overall, 111 items passed the first round with a $\mathrm{W}>0.7,10$ items did not reach the cut-off for agreement ( $\geq 70 \%)$ (Tables $1-4)$.

\section{Round 2}

Nineteen out of 20 panellists (95\%) evaluated the 111 items with a $\mathrm{W}>0.7$ and confirmed the agreement reached during round 1. Among the ten items to be re-voted, two items $(4.5,6.3)$ were not approved after the second round.

\section{Final criteria list}

\section{Panel synthesis for the suggestions}

Out of 121 suggestions, 119 received a high level of agreement. Tables 1 to 4 outline all final Practice Suggestions for each identified issue. Comments, justifications and highlights about each suggestion by authors and panellists are reported in the Appendix (appendix references 1-193). Two suggestions reached an unclear level of agreement $(\mathrm{W}<0.7)$.

Table 1. Personnel protection equipment.

\begin{tabular}{|c|c|c|c|c|}
\hline \multirow[t]{2}{*}{ Topics and recommendations } & \multicolumn{3}{|c|}{ Level of concordance } & \\
\hline & Low & Unclear & High & \\
\hline 1. Suggestions for personal protection needs & $\downarrow$ & $\rightleftarrows$ & $\uparrow$ & \\
\hline $\begin{array}{l}\text { 1.1 Healthcare professionals treating COVID-19 patients should wear appropriate personal protective } \\
\text { equipment (PPE) and they should be trained in how to put on and take off PPE to avoid self-contamination }\end{array}$ & $0.0 \%$ & $5.0 \%$ & $95.0 \%$ & Approved \\
\hline $\begin{array}{l}\text { 1.2 During the first } 3 \text { months after infection, also if patient has negative nasal/throat swabs, use eye and } \\
\text { respiratory protections, gloves and if possible disposable gown when aerosol generating procedures } \\
\text { (AGPs) are used }\end{array}$ & $5.0 \%$ & $5.0 \%$ & $90.0 \%$ & Approved \\
\hline 1.3 All patients should wear a medical mask, when possible, during treatment & $0.0 \%$ & $0.0 \%$ & $100.0 \%$ & Approved \\
\hline 1.4 Strategies to minimize dispersion of infected droplets and aerosol should be employed, during AGPs & $0.0 \%$ & $5.0 \%$ & $95.0 \%$ & Approved \\
\hline $\begin{array}{l}\text { 1.5 For outpatient consultation, the examination room should be aerated after each consultation and } \\
\text { surfaces have to be sanitized. In waiting room ensure spatial distance between patients }\end{array}$ & $0.0 \%$ & $10.0 \%$ & $90.0 \%$ & Approved \\
\hline
\end{tabular}




\section{Discussion}

There is no data about PR for COVID-19 after the acute phase. As no sufficient information about the long-term effects, extent of damage or sequelae after this disease were known at the time of the review, suggestions presented in this report are based on the definition of PR and its effectiveness in other chronic pulmonary diseases, on authors' and panelists' literature review and their recent experience with COVID-19 patients.

Keeping with single hospital policies of infection prevention and control, the use of PPE [9] is mandatory for all interventions. Panelists' care was focused on health-workers training, strategies to minimize droplets dispersion and to ensure the clearing of surfaces and devices.

The panel suggested that phenotyping could guide the timing for PR identifying patients which might benefit from an early program after discharge. Given the impact of the large number of affected patients on the healthcare team, hospital facilities and resource utilization should be used with a criteria-based enrolment. As exercise is the main trigger for dyspnea, fatigue and oxygen desaturation, adapted exercise testing for physical and respiratory muscles performance is recommended. Nevertheless, authors and most of the panelist agreed that "severe functional impairment should not be considered as a contraindication for PR". Similar to patients recovering from ARDS due to severe influenza A (H1N1) [10], those with an acute COVID-19 event may have functional damage (respiratory function, critical illness myopathy and neuropathy), reduced health related quality of life, in both the short- and long-term following discharge. The expected recovery time in such patients might vary depending upon the degree of acute respiratory failure and the associated physical (asthenia, peripheral muscle weakness, nutritional status) and emotional dysfunction (anxiety, depression, sense of abandonment, post-traumatic stress syndrome) as previously reported in post-ARDS patients [11]. The experts and panelists also emphasized the potential impact on outcomes of comorbidities (mainly neurological and cardiological). Moreover, the measure of ADL impairment at home and the role of caregivers' availability have been underlined as crucial for recovery. While CT scan was recognized as the gold standard to follow-up the pulmonary sequelae, the suggestion "Chest X-ray should be performed early within 3-5 months" was quoted as unclear, because it would not alter the approach to PR and no change were expected after a PR program. Timing and modality to perform arterial blood gases analysis and lung function tests were deeply discussed.

Suggestions have focused on type, intensity, timing and modalities of interventions to be tailored to the individual patient's needs. Pronation strategies [12] were included as part of PR programs for selected patients. Airway clearance techniques were recommended to be continued only for hypersecretive patients. Oxygen, at rest and during efforts, aerosol-therapy and drug-inhalers must be delivered with caution and adaptation to avoid droplet dispersion. As the effects of muscle activity in viral infections is not known, panelists suggested aerobic low load exercise based on subjective symptoms with low intensity $(<3.0 \mathrm{METs})$, together with daily patient counselling and education.

Table 2. Diagnosis of COVID-19 phenotype patients.

\begin{tabular}{|c|c|c|c|c|}
\hline Topics and recommendations & & of conco & ance & \\
\hline & Low & Unclear & High & \\
\hline 2. Phenotypes & $\downarrow$ & $\rightleftarrows$ & $\uparrow$ & \\
\hline $\begin{array}{l}\text { 2.1 Days of contagious risk, need of pulmonary rehabilitation (PR), timing to start PR and predictors of } \\
\text { recovery are unknown }\end{array}$ & $0.0 \%$ & $15.8 \%$ & $84.2 \%$ & Approved \\
\hline $\begin{array}{l}\text { 2.2 PR programs should be proposed to dyspnoeic, older, comorbid patients with long length of stay, } \\
\text { intensive care unit (ICU) history, needing weaning from mechanical ventilation (MV) or tracheostomy } \\
\text { cannula, reduced strength and exercise capacity, requiring oxygen at rest and during effort with lung } \\
\text { function and psychological impairment }\end{array}$ & $0.0 \%$ & $5.2 \%$ & $94.7 \%$ & Approved \\
\hline 2.3 Due to different conditions and patients' phenotypes, individualized programs should be proposed & $0.0 \%$ & $15.7 \%$ & $84.2 \%$ & Approved \\
\hline 2.4 The baseline assessment core set is not yet available & $0.0 \%$ & $21.1 \%$ & $78.9 \%$ & Approved \\
\hline 2.5 In case of tracheostomy, standardized protocols for cannula removal, swall & $0.0 \%$ & $5.2 \%$ & $94.7 \%$ & Approved \\
\hline
\end{tabular}

aspirations and decannulation are welcomed

3. Are frailty measurements important?

3.1 It is reasonable that patients with frailty are the most vulnerable to COVID-19

3.2 Patients with frailty could be affected by COVID-19 more seriously and developed a poor prognosis

3.3 Frailty should be early recognized before setting up the PR program, to reduce risk for poor COVID-19 outcomes

3.4 Frailty measurements should be integrated by multidimensional evaluation focusing on global exercise capacity, strength, balance, coordination, nutritional and psychosocial status.

4. Tliming of Pulmonary Rehabilitation start

4.1 There is currently no clear scientific evidence for the timing

4.2 PR must start early in the course of hospital treatment

4.3 PR must start already in the ICU to obtain the maximum benefits

\begin{tabular}{cccc}
$\downarrow$ & $\rightleftarrows$ & $\uparrow$ & \\
$0.0 \%$ & $11.1 \%$ & $88.9 \%$ & Approved \\
$0.0 \%$ & $11.1 \%$ & $88.9 \%$ & Approved \\
\hline $0.0 \%$ & $5.2 \%$ & $94.7 \%$ & Approved
\end{tabular}

4.4 Pulmonologist expert in rehabilitation field should coordinate the multidisciplinary team

4.5 Programs in outpatients and telemedicine should be considered for mild COVID-19 patients and patients discharged from hospitals

$0.0 \% \quad 5.2 \% \quad 94.7 \% \quad$ Approved

. $5.2 \% \quad 94 \%$ Approved

\begin{tabular}{cccc}
$0.0 \%$ & $22.2 \%$ & $77.7 \%$ & Approved \\
$0.0 \%$ & $11.1 \%$ & $83.3 \%$ & Approved \\
\hline $0.0 \%$ & $5.5 \%$ & $94.4 \%$ & Approved \\
$5.5 \%$ & $11.1 \%$ & $83.3 \%$ & Approved \\
\hline
\end{tabular}

$11.7 \% \quad 23.5 \% \quad 64.7 \% \quad$ Not Approved


Table 3. Assessments.

$\downarrow \quad \rightleftarrows$

5.1 The ability to predict discharge outcomes following COVID-19 is unknown

5.2 A complete resolution of the damage due to COVID-19 is probably possible for the most part of the patients, but it is not known how many patients will have irreversible of progressive damage

5.3 The role of comorbidities, severity of imaging features, laboratory data, in view of a successful discharge $\quad 0.0 \% \quad 17.6 \% \quad 82.4 \% \quad$ Approved are unknown

5.4 Symptoms scales, infectious disease/immunological status, haematological data, imaging, cardiorespiratory function, pulmonary function tests, respiratory muscle strength, nutritional status, comorbidities should be assessed

5.5 Neurological and psychological disorders (anxiety, depression) and frailty should be assessed

5.6 Exercise tolerance, functional status and physical performance, presence of critical illness neuromyopathy and ICU acquired weakness should be considered as an outcome measure

5.7 Activities of daily living (ADL), baseline functional impairment due to dyspnea and how breathlessness affects patient's mobility should be considered as an outcome measure

5.8 Role of caregiver, the availability of internet, the presence of tele-rehabilitation platform and the availability of rehabilitative home service should be assessed before discharge

\section{Which imaging is informative to individualize the program?}

6.1 Early changes of lung imaging by computed tomography (CT) scan toward consolidation are described within 15 days from admission

6.2 Chest X-ray may be useful to target individual interventions, but not a good outcome measure for the PR program

6.3 Chest X-ray should be performed early (3-5 months) in the follow-up

\section{When and how to assess gas exchanges? What are the best informative indexes?}

7.1 Blood gas analysis (ABG) with the PaO2/FiO2 values are the gold standard to measure gas exchanges

7.2 ABG is mandatory at admission and discharge with supplementary controls in case of severe dyspnea or fever

7.3 Pulse oximetry and $\mathrm{SaO} 2 / \mathrm{FiO} 2$ values are fundamental instrument for monitoring clinical situation at rest and during effort

7.4 Pulse oximetry device during self-managed at home is recommended

8. When and which lung function tests? $\downarrow$

8.1 Lung function tests may be proposed when safe to perform by operators and patients

8.2 Spirometry and diffusion capacity (DLCO) should be the gold standard being abnormal in 15\% and 50\% of cases

8.3 Severe impairment should not be considered as a contraindication for PR

8.4 Lung function tests are not outcome measures of programs

9. Functional evaluation (static and dynamic) - exercise capacity tests muscles measures tests

9.1 Before starting the rehabilitation program and at hospital discharge an assessment of physical performance and ADL autonomy is recommended; if abnormal values are found, further specific measures should be administered to quantify single limitations; these measures could be also used as rehabilitative outcome measures

9.2 Standard maximal Cardiopulmonary Exercise Test is not recommended in the first 6-8 weeks after acute hospital discharge due to unknown cardiorespiratory and muscle involvement and infectious risk

9.3 The assessment of exercise-induced oxygen desaturation is mandatory during the execution of exercise $\quad 0.0 \%$ tolerance tests calculating the in change in SpO2 during test (mean exercise - basal level)

9.4 During exercise tests and exercise sessions, fatigue and dyspnea should be assessed though psychometric scale (i.e. BORG scale or Visual Analogic Scale)

9.5 Because we expected different trajectory of exercise performance recovery, the monitoring of physical $\quad 0.0 \%$ performance should be routinely included in the follow-up assessment

$5.5 \% \quad 11.1 \% \quad 83.3 \% \quad$ Approved

$0.0 \% \quad 11.1 \% \quad 88.9 \% \quad$ Approved

$0.0 \% \quad 16.7 \% \quad 83.3 \% \quad$ Approved

(20)

$0.0 \% \quad 16.7 \% \quad 83.3 \% \quad$ Approved

$0.0 \% \quad 5.6 \% \quad 94.4 \% \quad$ Approved

$0.0 \% \quad 22.2 \% \quad 77.8 \% \quad$ Approved

$0.0 \% \quad 6.3 \% \quad 93.8 \% \quad$ Approved

$\downarrow \quad \rightleftarrows$

$\rightleftarrows$

$0.0 \% \quad 27.2 \% \quad 72.2 \% \quad$ Approved

0\% $\quad 11.1 \% \quad 88.8 \% \quad$ Approved

$17.6 \% \quad 23.5 \% \quad 58.8 \% \quad$ Not approved

$\downarrow \quad \uparrow$

$0.0 \% \quad 0.0 \% \quad 100.0 \% \quad$ Approved

$0.0 \% \quad 16.6 \% \quad 83.3 \% \quad$ Approved

$0.0 \% \quad 0.0 \% \quad 100.0 \% \quad$ Approved

$0.0 \% \quad 16.7 \% \quad 83.3 \% \quad$ Approved

$\rightleftarrows \quad \uparrow$

$0.0 \% \quad 0.0 \% \quad 100.0 \% \quad$ Approved

$0.0 \% \quad 5.9 \% \quad 94.1 \% \quad$ Approved

$6.5 \% \quad 0 \% \quad 93.7 \% \quad$ Approved

$0.0 \% \quad 0.0 \% \quad 100.0 \% \quad$ Approved

$\downarrow \quad \uparrow$

$0.0 \% \quad 11.1 \% \quad 88.9 \% \quad$ Approved


Table 3. Continued from previous page.

$\begin{array}{cc}\text { Topics and recommendations } & \text { Level of concordance } \\ \text { Low Unclear High }\end{array}$

10. Respiratory muscle assessment?

10.1 Prevalence, severity and recovery of respiratory muscle weakness due to COVID-19 are unknown, as well as their impact on symptoms and disability

10.2 Standard maximal inspiratory and expiratory pressures (MIP/MEP) are not recommended in the first phase (6-8 weeks) due to infectious risk. When performed, special PPE should be worn and antiviral filter should be placed between mouth/tracheostomy and devices, in order to limit contamination

10.3 In infectious patients, alternative modalities for MIP/MEP measurements using disposable devices, or alternative tests (ie. Single Breath Counting) could be used

10.4 MIP/MEP or surrogate measures may be used to set up a respiratory muscle-training program when $\quad 5.5 \% \quad 11.1 \% \quad 83.3 \% \quad$ Approved respiratory muscle weakness is speculated

$\downarrow \quad$ से

10.5 During the weaning of mechanical ventilation and/or tracheo-cannula, respiratory muscles strength tests $\quad 0.0 \% \quad 17.6 \% \quad 82.4 \% \quad$ Approved are recommended. The measurements should preferably be performed at the cannula (highest value)

$\mathbf{1 0 . 6}$ In mechanically ventilated patients, the estimation of inspiratory muscle strength may be performed $\quad 6.7 \% \quad 6.7 \% \quad 86.6 \% \quad$ Approved through ventilator using MIP and P 0.1 assessment

\section{Is secretion encumbrance a problem? How to assess the need for intervention?}

11.1 Assessment of mucus encumbrance or expectoration difficulties should be considered in all patient reporting pre-existing hyper-secretive condition, those after extubation or weaning from mechanical ventilation, those reporting phlegm or sticky mucus and productive cough

11.2 Anamnestic data, quantity and quality of expectorated mucus, lung sound auscultation and reported $\quad 0.0 \% \quad 11.1 \% \quad 88.8 \% \quad$ Approved symptoms should be considered to assess the need for an airway clearance augmentation strategy

11.3 SpO2 measure is not directly related to airway obstruction and mucus encumbrance, however could be $\quad 0.0 \% \quad 11.1 \% \quad 88.8 \% \quad$ Approved an adjunctive informative measure to test the efficacy of airway clearance manoeuvres

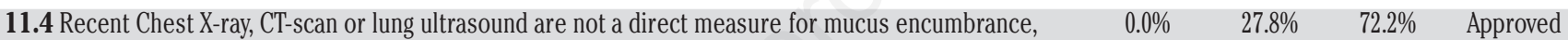
but could be informative about areas at risk of airway clearance impairment

12. How the nutritional status can affect functional recovery?

12.1 It is relevant to evaluate the nutritional status of patients hospitalized for moderate, severe and very $\quad 0.0 \% \quad 0.0 \% \quad 100.0 \% \quad$ Approved severe COVID-19 infection

12.2 The severe inflammation, the resulting hypercatabolic state and the drastic reduction of food intake $\quad 0.0 \% \quad 0.0 \% \quad 100.0 \% \quad$ Approved makes these patients at risk of malnutrition

12.3 Dysphagia screening has to be implemented at the same time as nutritional screening

12.4 It is important to implement a prompt and adequate nutritional assistance.

12.5 If dysphagia occurs, it must be promptly treated

13. How to assess quality of life ( $\left.Q_{0} \mathrm{~L}\right)$ and participation during social distancing, quarantine and isolation?

13.1 To evaluate the quality of life (QoL) it would be appropriate to test the presence of psychopathological $\quad 0.0 \% \quad 6.3 \% \quad 93.8 \% \quad$ Approved disorders (in particular anxiety, depression, sleep disturbance, post-traumatic stress disorder)

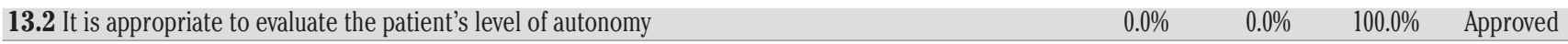

$\mathbf{1 3 . 3}$ It is appropriate to evaluate the quality of the support network (communication possibilities $\quad 0.0 \% \quad 11.7 \% \quad 88.2 \% \quad$ Approved of the patient, stress of the caregiver)

13.4 It is appropriate to have a global measurement of the patient's perceived QoL level $\quad 0.0 \% \quad 5.6 \% \quad 94.4 \% \quad$ Approved

14. How to identify emotional aspects influencing participation to PR program?

14.1 A neuropsychological assessment should be performed at baseline and after PR

14.2 Psychosocial effects (such as depression, anxiety, psychosomatic preoccupations, insomnia) should be measured

14.3 Symptoms of Post-Traumatic Stress Disorder (PTSD) should be considered

$11.8 \% \quad 11.8 \% \quad 76.5 \% \quad$ Approved

14.4 The long-term psychological and psychosocial implications of infectious diseases should not be ignored

14.5 A peculiar attention should be played to caregiver and family of those in quarantine because affected by COVID-19 
A well-structured multidisciplinary follow-up program was strongly recommended. Telemedicine is certainly a suggestive opportunity to continue following patients by reducing travel for the patient and reducing the risk of contagion for operators, mainly in those patients who suffer from exercise dyspnea. Although this hypothesis was proposed, interestingly, experts quoted the use of telerehabilitation with unclear level of agreement. Reasons were the unclear definition of "mild" COVID, the inconclusive literature evidence, the "prevalent belief" that telerehabilitation had to be proposed only in stable patients. The use of information communication technology in this frame is interesting [13] but obstacles must be completely overcome: i) the technology must be usable by the largest possible number of patients; ii) patients must be treated at home ensuring maximum safety; iii) clarity on medico-legal liability; iv) adequate evaluation of the economic reimbursement.
This document has several strengths. It responds to an urgent need for clinical guidance for professionals, and stakeholders worldwide. Guidance was based on the most recent and relevant COVID-19 clinical practice guidelines [14] from highly respected, national physiotherapy organizations and peer-reviewed studies; these sources were transparently reported. The authors represent an international group of physicians and physiotherapists, with extensive experience in PR.

There are also some limitations. Given the recent presentation of COVID-19, clinical guidance may change as more is learnt about the natural history of disease. Suggestions were extrapolated based on best evidence for current management of post-COVID-19 patients with rehabilitation programs. No patient was included in the author group. COVID-19 is placing significant demands on healthcare resources throughout the world.

Table 4. Interventions.

$\begin{array}{cc}\text { Topics and recommendations } & \text { Level of concordance } \\ \text { Lnclear High }\end{array}$

15. How to manage oxygen-therapy and interface? How to dose and scale oxygen at rest and during physical activity?

15.1 Oxygen need at rest, during effort and sleep should be assessed before setting up the program $\quad 0.0 \% \quad 11.1 \% \quad 88.9 \% \quad$ Approved

15.2 Suitable interface (in term of efficacy and patient tolerance) should be tested before setting up $\quad 0.0 \% \quad 5.6 \% \quad 94.4 \% \quad$ Approved the program

15.3 Oxygen need during effort should be assessed through standardized tests (6-minute walk test or other $\quad 0.0 \% \quad 0.0 \% \quad 100.0 \% \quad$ Approved field tests) and reassessed during the PR program based on exercise progression

15.4 Specific precautions about the exhaled air dispersion distance should be taken into account during $\quad 0.0 \% \quad 5.6 \% \quad 94.4 \% \quad$ Approved oxygen administration

16. Exercise programs

16.1 PR in post-acute COVID-19 could improve symptoms, functional capacity and quality of life; however, $\quad 0.0 \% \quad 0.0 \% \quad 100.0 \% \quad$ Approved the best exercise program is still unknown.

16.2 The exercise training principles used in patients with chronic lung diseases can be considered also $\quad 0.0 \% \quad 18.8 \% \quad 81.3 \% \quad$ Approved in post-COVID-19 patients

16.3 Aerobic exercise $<3.0$ METs with progressive increase of intensity based on symptoms (BORG fatigue $\quad \begin{array}{llll}0.0 \% & 0.0 \% & 100.0 \% & \text { Approved }\end{array}$ and/or dyspnea below the score of 3 ) is recommended in patients with mild or no disability (SPPB >10; Barthel index $>70$ ) in order to restore a normal physical function 16.4 Patients with moderate or severe disability $(\mathrm{SPPB}<10$; Barthel index $<70)$ need a comprehensive
pulmonary rehabilitation program in order to improve autonomy, peripheral and respiratory muscle strength, balance, walking ability, symptoms and quality of life

16.5 The exercise program should include aerobic exercises (cycling, treadmill, free walking) and $\quad$ 0.0\% $\quad 5.8 \% \quad 94.1 \% \quad$ Approved resistance strength training

16.6 SpO2 monitoring during exercise is mandatory and subsequent oxygen supplementation could be $\quad 5.8 \% \quad$ 11.8\% $\quad 82.4 \% \quad$ Approved prescribed when $\mathrm{SpO} 2<93 \%$, being aware of potential environmental contamination

16.7 Non-invasive ventilation during exercise training should be used with specific cautions to avoid the risk $\quad 22.2 \quad 5.5 \% \quad 72.2 \% \quad$ Approved of environmental contamination.

16.8 In case of tracheostomy, the use of speaking valve during exercise should be preferred to open $\quad 0.0 \% \quad 6.3 \% \quad 93.8 \% \quad$ Approved HME filters

17. When and which lung recruitment exercises? Which strategies and devices?

17.1 Individualized recruitment strategies such as chest expansion breathing control exercises associated to posture positioning should be considered as part of PR program

17.2 Posture positioning should be chosen in according to chest X-ray/CT scan (if any), auscultation, SpO2 change and patient reported symptoms

17.3 Continuous or temporary positive expiratory pressure (PEP, TPEP) devices, also including visual or acoustic feedback, should be considered, alone or in combination with posture

17.4 An inspiratory flow-dependent resistance can be used to slow down inspiratory flow and to increase $\quad 6.7 \% \quad 20.0 \% \quad 73.33 \% \quad$ Approved inspiratory time, enhancing pleural traction on peripheral lung regions 
Table 4. Continued from previous page.

$\begin{array}{cc}\text { Topics and recommendations } & \text { Level of concordance } \\ \text { Low Unclear High }\end{array}$

18.1 Aerosol/Nebulizer treatment administration is NOT recommended

18.2 If patient is mechanically ventilated, inhalation therapy should be administered during mechanical ventilation, using metered-dose inhalers (MDI) or ultrasonic nebulizers connected to the mechanical ventilator in a closed circuit, without removing the antimicrobial filter on the expiratory branch of the circuit

18.3 To deliver inhaled therapy during mechanical ventilation, the use of MDI or ultrasonic nebulizers connected to the mechanical ventilator in a closed circuit is recommended, without removing the antimicrobial filter on the expiratory limb of the circuit

18.4 If bronchodilation is needed, MDI with spacer or dry powder inhaler (DPI) should be considered.

18.5 DPIs are preferred if patient's inspiratory capacity is sufficient to activate the inhaler

\section{When and which strategies and devices for bronchial hygiene?}

19.1 Airway clearance augmentation strategies and techniques (ACTs) should be continued, with adaptation $\quad 0.0 \% \quad 12.5 \% \quad 87.5 \% \quad$ Approved if needed, in chronic hypersecretive patients and should be considered for subject experiencing phlegm and/or productive cough

19.2 In hypersecretive patients, the use of continuous or temporary positive expiratory pressure devices, with or without oscillation, (PEP, TPEP, OPEP) should be considered, alone or in combination with lung expansion strategies, to enhance lung volume recruitment, to better control the expiration flow and to facilitate peripheral and proximal mucus mobilization.

19.3 Flow-dependent low resistance positive expiratory pressure (PEP) systems, with an antibacterial filter on expiration circuit, are more tolerated and should be preferred to high resistance and threshold-PEP, mostly in weaker or symptomatic patients

19.4 Since cough is one of the most annoying symptoms in COVID-19 lung involvement and can cause dyspnea or chest pain, forced expiratory flows (huffs) should be preferred to expectorate

19.5 Among ACTs, those that enable patient to auto-treatment should be preferred

\begin{tabular}{llll}
$11.1 \%$ & $0 \%$ & $88.8 \%$ & Approved \\
& & & \\
\hline $6.6 \%$ & $6.6 \%$ & $86.6 \%$ & Approved
\end{tabular}

19.6 Jet/mesh nebulizer (with filters on the exhalation port and mouthpiece) and humidification should be considered in association to airway clearance intervention

19.7 During invasive mechanical ventilation suctioning should be performed with a closed suction system $\quad 5.9 \% \quad 0.0 \% \quad 94.1 \% \quad$ Approved and an in-line viral filter

20.1 Respiratory muscle training is not recommended routinely, but it should be administered whenever respiratory muscle weakness is detected, particularly in patients candidate to decannulation or with persistent dyspnea

20.2 The type, efficacy and duration of respiratory muscle training in COVID-19, either in the post-acute phase or in the long-term at patient's home, has still to be investigated

20.3 The inspiratory muscle training should be started at low intensity. The progression must be guided by dyspnea/fatigue and by the monitoring of vital signs

$0.0 \% \quad 18.8 \% \quad 81.3 \% \quad$ Approved

$11.7 \% \quad 11.7 \% \quad 76.4 \% \quad$ Approved

$5.5 \% \quad 5.5 \% \quad 88.8 \% \quad$ Approved

\begin{tabular}{cccc}
$5.5 \%$ & $5.5 \%$ & $88.8 \%$ & Approved \\
\hline $0.0 \%$ & $6.3 \%$ & $93.8 \%$ & Approved \\
$\downarrow$ & $\rightleftarrows$ & $\uparrow$ & \\
$0.0 \%$ & $12.5 \%$ & $87.5 \%$ & Approved
\end{tabular}

\begin{tabular}{cccc}
$0.0 \%$ & $13.3 \%$ & $86.7 \%$ & Approved \\
& & & \\
\hline $0.0 \%$ & $6.7 \%$ & $93.3 \%$ & Approved \\
$14.2 \%$ & $14.2 \%$ & $71.4 \%$ & Approved \\
& & & \\
\hline $5.9 \%$ & $0.0 \%$ & $94.1 \%$ & Approved
\end{tabular}

20.4 MIP/MEP or surrogate measures should be considered as main outcome measures for respiratory muscle training

20.5 Respiratory muscle training should be performed using disposable dedicated devices

21. Is tele-coaching/tele-monitoring/telerehabilitation possible, effective and safe for these patients?

21.1 Tele-rehabilitation (TR) could represents the appropriate response in the post-acute phase by combining need of PR with need for social distancing

21.2 TR may allow to increase the accessibility of PR eliminating issues of transport, travel, their associated $\quad 0.0 \% \quad 6.7 \% \quad 93.3 \% \quad$ Approved costs and weather

21.3 TR should be adopted in patients with mild to moderate disabilities needs for frequent monitoring, with residual disability after PR residing in isolated areas or without availability of standard PR program

21.4 Vital parameters (SpO2, heart rate, blood pressure, respiratory rate) as symptoms should be recorded before the start of the telerehabilitation intervention and then monitored daily

21.5 Proper training of health professionals involved and the verification of the technological requirements, especially at the patient's home, are required

21.6 Adequate caregiver support could be necessary in case of residual disability or for technological setting up

$5.9 \% \quad 11.8 \% \quad 82.4 \% \quad$ Approved

\begin{tabular}{llll}
$0.0 \%$ & $5.9 \%$ & $94.1 \%$ & Approved \\
\hline $6.3 \%$ & $18.8 \%$ & $75.0 \%$ & Approved
\end{tabular}

$11.7 \% \quad 11.7 \% \quad 76.5 \% \quad$ Approved

$12.5 \% \quad 6.3 \% \quad 81.3 \% \quad$ Approved

$\downarrow \quad \uparrow$


Table 4. Continued from previous page.

\begin{tabular}{|c|c|c|c|c|}
\hline \multirow[t]{2}{*}{ Topics and recommendations } & \multicolumn{3}{|c|}{ Level of concordance } & \\
\hline & Low & Unclear & High & \\
\hline $\begin{array}{l}\text { 22. When and what kind of re-assessment is recommended? When a multidisciplinary } \\
\text { follow-up is required? In which setting? }\end{array}$ & $\downarrow$ & $\rightleftarrows$ & & \\
\hline $\begin{array}{l}\text { 22.1 The reassessment should be performed at the end of the post-acute phase, before the transfer to } \\
\text { another location (rehabilitation institute for intensive respiratory rehabilitation or home) and therefore } \\
\text { every } 3 \text { months for } 1 \text { year in more severe cases }\end{array}$ & $0.0 \%$ & $0.0 \%$ & $100.0 \%$ & Approved \\
\hline $\begin{array}{l}\text { 22.2 The setting after the post-acute phase have should be chosen based on the characteristics of the } \\
\text { patients. A hospital setting (rehabilitation institute for intensive rehabilitation) can be indicated in } \\
\text { patients with 1) tracheostomy, CPAP or BIPAP therapy, oxygen therapy at rest 2) extra-pulmonary } \\
\text { comorbidities or severe disability with lack of autonomy in the activities of daily life. A home setting can be } \\
\text { indicated in patients with sufficient autonomy, adequate home support, mild disability, one or } \\
\text { no comorbidity, no need for monitoring }\end{array}$ & $0.0 \%$ & $11.8 \%$ & $88.2 \%$ & Approved \\
\hline $\begin{array}{l}\text { 22.3 Follow-up by a multidisciplinary team is recommended in patients with critical and severe disease, } \\
\text { extrapulmonary manifestations of COVID-19 and in those with past disabilities, in order to evaluate } \\
\text { their evolution over time }\end{array}$ & $0.0 \%$ & $5.9 \%$ & $94.1 \%$ & Approved \\
\hline $\begin{array}{l}\text { 23. What are the risks and benefits of exercise training in COVID-19 patients with } \\
\text { cardiovascular complications? }\end{array}$ & $\downarrow$ & $\rightleftarrows$ & & \\
\hline 23.1 During exercise training ECG, automatic blood pressure and $\mathrm{SpO} 2$ monitoring is recommended & $5.7 \%$ & $16.6 \%$ & $77.7 \%$ & Approved \\
\hline $\begin{array}{l}\text { 23.2 Supplementary monitoring for symptom check (BORG for dyspnea and Rate of Perceived Exertion } \\
\text { scale RPE) are useful }\end{array}$ & $0.0 \%$ & $6.3 \%$ & $93.8 \%$ & Approved \\
\hline 23.3 Effort tolerance, strength measurements, ADL, inflammatory indices are useful outcomes parameters & $0.0 \%$ & $0.0 \%$ & $100.0 \%$ & Approved \\
\hline $\begin{array}{l}\text { 23.4 If home programs are proposed a hybrid administration where the evaluation is carried out in person, } \\
\text { and supervision of the exercise training program remotely may be the optimal solution }\end{array}$ & $0.0 \%$ & $6.7 \%$ & $93.3 \%$ & Approved \\
\hline
\end{tabular}

PPE, personal protective equipment; AGPs, aerosol generating procedures; PR, pulmonary rehabilitation; ICU, intensive care unit; MV, mechanical ventilation; $\mathrm{ADL}$, activities of daily living; CT, computed tomography; ABG, blood gas analysis; PaO2/FiO2, ratio of arterial oxygen partial pressure to fractional inspired oxygen; SaO2/FiO2 ratio of oxygen saturation to fractional inspired oxygen; DLCO, diffusing capacity for carbon monoxide; MIP/MEP, maximal inspiratory and expiratory pressures; P 0.1, airway occlusion pressure; SpO2, peripheral capillary oxygen saturation; QoL, quality of life; PTSD, post-traumatic stress disorder; METs, metabolic equivalents; SPPB, short physical performance battery; HME, heat and moisture exchanger; PEP, positive expiratory pressure; TPEP, temporary positive expiratory pressure; MDI, metered-dose inhalers; DPI, dry powder inhaler ACTs, airway clearance techniques; OPEP, oscillating positive expiratory pressure; TR, tele-rehabilitation; CPAP, continuous positive airway pressure; BIPAP, bilevel positive airway pressure; ECG, electrocardiogram.

In conclusion, the dramatic spread of the pandemic with its related clinical consequences and socio-economic impact is driving the development of clear and effective measures to reduce the dramatic impact on patients and their families. Healthcare facilities must have the capacity and flexibility to respond with the higher available healthcare expertise to the needs of all patients, in the most appropriate locations using the most modern technology. As with so many primarily respiratory conditions, pulmonary rehabilitation has an important role to play in promoting recovery and improving community reintegration by increasing mobility, autonomy and health related quality of life.

\section{References}

1. Lian J, Jin X, Hao S, et al. Analysis of epidemiological and clinical features in older patients with corona virus disease 2019 (COVID-19) out of Wuhan. Clin Infect Dis 2020. doi: $10.1093 / \mathrm{cid} / \mathrm{ciaa} 242$.

2. Gandotra S, Lovato J, Case D, et al. Physical function trajectories in survivors of acute respiratory failure. Ann Am Thorac Soc 2019; 16:471-7.

3. Spagnolo P, Balestro E, Aliberti S, et al. Pulmonary fibrosis secondary to COVID-19: a call to arms? Lancet Respir Med 2020. doi: 10.1016/S2213-2600(20)30222-8.

4. Fitch K, Bernstein SJ, Aguilar MD, et al. The RAND/UCLA appropriateness method user's manual. Santa Monica: RAND Corporation; 2001.

5. Moher D, Schulz KF, Simera I, Altman DG. Guidance for developers of health research reporting guidelines. PLoS Med 2010;7:e1000217.

6. Arksey H, O'Malley L. Scoping studies: towards a methodological framework. Int J Soc Res Methodol 2005;8:19-32.

7. WHO. Coronavirus disease (COVID-19) pandemic. Available from: https://www.who.int/

8. Beeching NJ, Fletcher TE, Fowler, R. Coronoavirus disease 2019 (COVID-19). BMJ Best Practices 2020. Accessed on: 25 March 2020. Available from: https://bestpractice.bmj.com/topics/en-gb/3000168.

9. Ippolito M, Vitale F, Accurso G, et al Medical masks and Respirators for the Protection of Healthcare Workers from SARS-CoV-2 and other viruses. Pulmonology 2020. doi: 10.1016/j.pulmoe.2020.04.009.

10. Luyt CE, Combes A, Becquemin MH, et al. Long-term outcomes of pandemic 2009 influenza A(H1N1)-associated severe ARDS. Chest 2012;142:583-92.

11. Herridge MS, Tansey CM, Matté A, et al. Functional disability 5 years after acute respiratory distress syndrome. N Engl J Med 2011;364:1293-304.

12. Longhini F, Bruni A, Garofalo E, et al. Helmet continuous positive airway pressure and prone positioning: A proposal for an early management of COVID-19 patients. Pulmonology 2020. doi: 10.1016/j.pulmoe.2020.04.014.

13. Mazzoleni S, Turchetti G, Ambrosino N. The COVID-19 outbreak: From "black swan" to global challenges and opportunities. Pulmonology 2020;26:117-8. doi: 10.1016/j.pulmoe.2020.03.002.

14. Vitacca M, Carone M, Clini EM, et al. Joint statement on the role of respiratory rehabilitation in the COVID-19 crisis: the Italian position paper. Respiration 2020. doi: 10.1159/ 000508399 . 